\title{
In vivo Monitoring and Assessment of Exogenous Mesenchymal Stem Cell-Derived Exosomes in Mice with Ischemic Stroke by Molecular Imaging
}

This article was published in the following Dove Press journal: International Journal of Nanomedicine

\section{Rong $\mathrm{Xu}^{*}$ \\ Yingying Bai* \\ Shudan Min \\ Xiaoxuan Xu \\ Tianyu Tang \\ Shenghong Ju}

Jiangsu Key Laboratory of Molecular and Functional Imaging, Department of Radiology, Zhongda Hospital, Medical School of Southeast University, Nanjing 210009, People's Republic of China

*These authors contributed equally to this work
Correspondence: Shenghong Ju Jiangsu Key Laboratory of Molecular and Functional Imaging, Department of Radiology, Zhongda Hospital, Medical School of Southeast University, 87

Dingjiaqiao Road, Nanjing 210009 ,

People's Republic of China

Tel +862583272121

Fax +862583311083

Email jsh0836@126.com
Purpose: Mesenchymal stem cell-derived exosomes (MSC-exos) are considered an important restorative treatment for ischemic stroke. However, the migration ability and survival of exogenous MSC-exos remain unclear. Here, we investigated whether MSC-exos migrate into the ischemic brain and play a protective role against ischemic stroke.

Methods: MSC-exos labeled with DiR were injected intravenously into mice with ischemic stroke. Near-infrared fluorescence (NIRF) images were obtained on days $0,1,3,5,7,10$, and 14 , and magnetic resonance (MR) images were obtained on days 1, 7 and 14. On day 14, the functional outcomes, angiogenesis, neurogenesis, and white matter remodeling were assessed, and Western blot assays were performed.

Results: Fluorescence signals from the MSC-exos appeared in the injured brain from day 1 and peaked on day 3 . The immunofluorescence staining of the brain samples revealed that the MSC-exos were localized in neurons. The behavioral scores and T2-weighted imaging indicated that the MSC-exos improved neurological functional recovery after stroke. In addition, the in vivo MR-diffusion tensor imaging (DTI) indicated that the exogenous MSCexos increased the fractional anisotropy (FA) value, fiber length, and fiber number ratio. Furthermore, in the mice with ischemic stroke treated with MSC-exos, angiogenesis and neurogenesis were significantly improved, and the expression of IL-1 $\beta$ was reduced.

Conclusion: MSC-exos can migrate into the brains of mice with ischemic stroke and exert therapeutic effects against ischemic stroke; therefore, MSC-exos may have broad clinical applications in the future.

Keywords: MSCs, exosomes, homing, near-infrared fluorescence, stroke, diffusion tensor imaging

\section{Introduction}

Stroke is the second leading cause of death worldwide and is characterized by high morbidity, disability and mortality. ${ }^{1}$ Ischemic stroke, which accounts for $80 \%$ of stroke cases in China, occurs when cerebral blood flow is insufficient in certain areas of the brain, leading to neurological dysfunction. ${ }^{2,3}$ Ischemic stroke seriously threatens human life and health and imposes a large burden on families. ${ }^{4}$ Currently, limited therapeutic options are available for ischemic stroke. Recombinant tissue plasminogen activator (rt-PA) is the only thrombolytic agent approved by the FDA and has a 4.5-h therapeutic window for the reversion of ischemic stroke. ${ }^{5,6}$ However, given the limited time window and risk of cerebral hemorrhage and reperfusion injury events associated with rt-PA, many patients still cannot achieve 
optimal treatment outcomes. ${ }^{7}$ Moreover, the blood-brain barrier (BBB) limits many beneficial agents from reaching the central nervous system at effective concentrations. ${ }^{3}$ Therefore, methods for overcoming these limitations are currently under investigation.

Mesenchymal stem cells (MSCs), which are capable of self-replication and self-differentiation, have emerged as promising candidates for cell therapy for many central nervous system diseases, including ischemic stroke. MSCs can secrete various functional products, such as cytokines, chemokines, bioactive factors and small molecular mediators, that can repair damaged tissues after ischemic stroke. ${ }^{8}$ Imaging studies of MSC migration in mice with ischemic stroke has demonstrated that MSCs can specifically home to injury sites within the brain. ${ }^{9}$ However, applying MSCs in ischemic stroke patients remains a challenge due to various factors, such as tumorigenicity, a high risk of embolism and difficulties in cell preservation. $^{10-12}$

Early studies suggested that MSCs enhance the structural and functional repair of damaged tissues by directly replacing damaged resident cells. ${ }^{13}$ However, currently, it is generally accepted that the paracrine effect of MSCs, rather than the effects of direct differentiation and replacement, is dominant in tissue regeneration. ${ }^{14}$ Moreover, many reports have recently suggested that MSC-exos, which are novel paracrine messengers, exert a positive effect in various damaged tissues, such as brain, kidney, heart, limb and bone tissues. ${ }^{15-20}$

Exosomes are nanosized extracellular vesicles that can be released by almost all cell types. ${ }^{19}$ Various cargos carrying important information from parent cells, such as proteins, lipids, peptides, DNA, RNA and bioactive factors, are packaged in exosomes. ${ }^{21}$ Exosomes participate in intercellular communication in a complex manner mainly through four pathways, including direct stimulation, endocytosis, membrane fusion and ligand-receptor binding; however, the mechanisms of action of exosomes remain unknown. ${ }^{22,23}$ MSCs are capable of mass-producing exosomes, rendering them ideal for cell-free therapy. Many studies have revealed that MSC-exos have the ability to improve functional recovery, accelerate vascular endothelial cell proliferation and decrease focal inflammation in a manner comparable to MSCs in mice with ischemic stroke. $^{24-27}$ While the homing pattern of MSCs in ischemic stroke has been investigated, whether MSCexos also retain the migration capacities of MSCs remains unknown. ${ }^{9}$
In addition, many reports have considered that exogenous MSC-exos can enhance white matter repair and promote neurite remodeling in a mouse model of stroke. ${ }^{25}$ Nevertheless, to date, few studies have assessed the structural changes and white matter remodeling in vivo after MSC-exo injection in ischemic stroke models by magnetic resonance imaging (MRI). DTI, which is a functional MRI sequence used for white matter lesion diagnosis, can describe the anisotropy of extracellular water molecules and the integrity of the microstructure. ${ }^{28,29}$ Fiber tracking using DTI enables the visualization of white matter pathways. ${ }^{29}$ Moreover, the FA value of DTI can be used to noninvasively measure the parameters of anisotropy of water diffusion, and a high value indicates that water diffusion is constrained in one dominant direction. ${ }^{29}$

Here, we aimed to understand the migration profile of MSC-exos in a mouse model of stroke after systemic infusion using near-infrared dye labeling, and using DTI combined with traditional methods, we assessed the roles of exogenous MSC-exos in white matter remodeling, functional recovery, angiogenesis, and neurogenesis.

\section{Materials and Methods \\ Cell Culture}

MSCs derived from the bone marrow of C57BL/6 mice were purchased from Cyagen Biosciences, Inc. (Guangzhou, China). ${ }^{30}$ The MSCs were cultured in $75 \mathrm{~cm}^{2}$ polystyrene tissue culture flasks (Corning, USA) at a density of $5 \times 10^{3}$ cells $/ \mathrm{cm}^{2}$ in DMEM/F12 medium (Hyclone, USA) supplemented with $10 \%$ fetal bovine serum (FBS) (Gibco, USA). The MSCs were passaged at a ratio of 1:3 using trypsin/EDTA solution (Hyclone, USA). All cultures were incubated in a humidified incubator containing $5 \% \mathrm{CO}_{2}$ at $37{ }^{\circ} \mathrm{C}$.

\section{Purification of Exosomes}

MSCs were cultured in medium supplemented with $10 \%$ exosome-depleted FBS prepared by centrifugation at $200,000 \times \mathrm{g}$ for $6 \mathrm{~h}$. After incubation for $48 \mathrm{~h}$, the culture supernatants were harvested for the exosome extraction. Briefly, the supernatants were centrifuged at $300 \mathrm{x} g$ for 10 minutes, $1200 \mathrm{x} \mathrm{g}$ for 20 minutes and $10,000 \mathrm{x}$ g for 30 minutes to remove cell debris and dead cells and then filtered through a $0.22 \mu \mathrm{m}$ filter to remove any large vesicles. After removing the precipitate, the supernatants were ultracentrifuged at $200,000 \mathrm{xg}$ for $2 \mathrm{~h}$ at $4{ }^{\circ} \mathrm{C}$, during which the exosomes were pelleted. Then, the exosome 
pellet was resuspended in PBS, and the suspension was ultracentrifuged as described above to obtain purified exosomes. The purified exosomes were stored at $-80{ }^{\circ} \mathrm{C}$.

\section{Characterization of Exosomes}

The exosome suspension was applied to a formvar carboncoated nickel grid. The grid was air-dried and stained with $2 \%$ uranyl acetate for $1 \mathrm{~h}$. Then, transmission electron microscopy (TEM) was performed to observe the shape and size of the exosomes. A nanoparticle tracking analysis (NTA) was conducted using a ZetaView nanoparticle tracking analyzer to evaluate the particle size distribution. In addition, the expression of tetraspanins (CD9 and CD63), which are specific exosome markers, was determined by a Western blot analysis.

\section{DiR-Labeled Exosomes}

Purified MSC-exos in PBS were incubated with $1 \mathrm{mM}$ DiR (Invitrogen, USA), which is a fluorescent lipophilic tracer, at room temperature (RT) for 30 minutes. Then, the labeled exosomes were separated from the unbound DiR by two rounds of ultracentrifugation as described above.

\section{Animals}

All animal experiments were approved by the Institutional Animal Care and Use Committee (IACUC) of the Medical School of Southeast University, according to Laboratory animal-Guideline for ethical review of animal welfare (GB/T 35,892-2018). Male C57BL/6J mice (25-27 g, 8-10 w) were purchased from Minhang Campus of East China Normal University (Shanghai, China) and adapted to the experimental conditions for 7 days before experimentation. A photothrombotic model of ischemic stroke was chosen because of the simple procedures and low death rate associated with this model. ${ }^{31}$ Briefly, the mice were anesthetized by an intraperitoneal injection of $1 \%$ pentobarbital $(50 \mathrm{mg} / \mathrm{kg})$. A depilatory cream was applied to the heads of the mice to expose the scalp. The mice were intraperitoneally injected with $1 \%$ rose Bengal $(100 \mathrm{mg} / \mathrm{kg})$, and after 5 minutes, a cold light source (Zeiss, USA) was applied $-2.0 \mathrm{~mm}$ anteroposterior and $2.0 \mathrm{~mm}$ mediolateral from bregma. The illumination lasted for 18 minutes.

\section{Near-Infrared Fluorescence Imaging (NIRFI)}

The in vitro and in vivo imaging of the DiR-labeled MSCexos was performed using an IVIS spectrum imager. On day 0 , images of the mice with stroke were captured as a baseline.
Then, DiR-labeled and unlabeled MSC-exos $(100 \mu \mathrm{g}, 200$ $\mu \mathrm{L}$ PBS) were administered to the mice through the tail vein. The mice were anesthetized with isoflurane and maintained in a prone position. Similarly, images were captured 1, 3, 5, 7, 10 and 14 days after stroke. The fluorescence signals in the tissues were quantified using IVIS software.

\section{Behavioral Tests}

The behavioral tests were performed on day 14 after stroke. The mice were placed on a grid-like platform and allowed to move freely for 5 minutes while a camera recorded from below. A foot fault was counted when the forelimb slipped through an opening in the grid. The numbers of foot faults and total steps were measured by watching the previously obtained video recordings. The foot fault percentage was calculated by determining the percentage of foot faults relative to the number of total steps. The modified neurological severity score (mNSS) $)^{32}$ test, which included four parts, namely, the motor function, sensation reflex, balance and abnormal action parts, was performed as described in previous studies. ${ }^{32}$ The details of mNSS test was presented in the supplementary material.

\section{Magnetic Resonance Imaging}

MRI was performed using a 7T small animal imaging scanner (Bruker, Germany). The heart rate of the mice was maintained at approximately $60 \mathrm{bpm}$ with isoflurane inhalation anesthesia. The T2-weighted imaging (T2WI) consisted of the following sequence: repetition time $=3 \mathrm{~s}$, echo time $=36 \mathrm{~ms}$, field of view $(\mathrm{FOV})=2 \times 2 \mathrm{~cm}$, number of axial slices $=15$, and slice thickness $=1 \mathrm{~mm}$. DTI was performed using the following echo-planar imaging sequence: reference images $=5$, repetition time $=5 \mathrm{~s}$, diffusion directions $=30$, echo time $=5 \mathrm{~ms}, \quad b$ value $=1000 \mathrm{~s} / \mathrm{mm}^{2}$, slices $=20$, slice thickness $=0.6 \mathrm{~mm}, F O V=20 \times 20 \mathrm{~mm}$, matrix size $=128 \times 128$, and total scan time $=23 \mathrm{~min}$ and 20s. The infarct volume was determined based on the T2WI images using ImageJ (National Institutes of Health, USA) software by researchers who were blinded to the groups. The fractional anisotropy (FA) value was evaluated using ParaVision 5.0 (Bruker, Germany) software. Fiber tracking was performed using a Diffusion Toolkit (version 0.6.4.1) and TrackVis (Version 0.6.1) software.

\section{Immunofluorescence}

The mice were perfused transcardially, and their brain tissues were immediately removed, placed in $4 \%$ paraformaldehyde for $48 \mathrm{~h}$ and subsequently placed in a $30 \%$ 
sucrose solution at $4{ }^{\circ} \mathrm{C}$ until they sank. Then, the brains were embedded with OCT (Sakura Finetek, USA) and stored at $-80{ }^{\circ} \mathrm{C}$. Ten-micrometer-thick sections of the mouse brains were acquired and blocked with bovine serum albumin (Biofroxx, Germany). The sections were incubated with a rabbit polyclonal anti-NeuN antibody (Abcam, UK) at $4{ }^{\circ} \mathrm{C}$ overnight and then incubated with an Alexa Fluor 546-conjugated goat anti-rabbit antibody (Abcam, UK) at RT for 90 minutes. The nuclei were stained with DAPI.

\section{Immunohistochemistry}

The brain samples were fixed as described above and embedded in paraffin at $-20^{\circ} \mathrm{C}$. The paraffin-embedded brains were sectioned into $4-\mu \mathrm{m}$ slices and incubated with a rabbit polyclonal anti-CD31 antibody (Abcam, UK), a rabbit polyclonal anti-Ki67 antibody (Abcam, UK), a rabbit polyclonal anti-maltose-binding protein (MBP) antibody (Abcam, UK), and a rabbit polyclonal anti-doublecortin (DCX) antibody (Abcam, UK) at $4{ }^{\circ} \mathrm{C}$. Then, the slices were examined under a microscope (Zeiss, Germany) by observers blinded to the groups.

\section{Western Blot Analysis}

The ipsilateral hemispheres of the mouse brain samples were collected and homogenized in whole cell lysis buffer
(KeyGEN BioTECH, China). The protein supernatants were centrifuged at $12,000 \mathrm{x}$ g for 10 minutes at $4{ }^{\circ} \mathrm{C}$ and denatured at $100{ }^{\circ} \mathrm{C}$. The proteins were subjected to SDS-PAGE (KeyGEN BioTECH, China) and transferred to PVDF membranes (Millipore, USA). Then, the membranes were blocked with 5\% skim milk and incubated with a rabbit polyclonal anti-interleukin $1 \beta$ (IL-1 $\beta$ ) antibody (Abcam, UK), a rabbit polyclonal anti-TNF $\alpha$ antibody (Abcam, UK), a rabbit polyclonal anti-brain-derived neurotrophic factor (BDNF) antibody (Abcam, UK) and a rabbit anti-GAPDH antibody (Abcam, UK) overnight at $4{ }^{\circ} \mathrm{C}$, followed by incubation with an HRP-linked secondary antibody (Cell Signaling Technology, USA) for $2 \mathrm{~h}$ at RT. Finally, the membranes were exposed and analyzed with ImageJ software.

\section{Statistical Analysis}

The data are represented as the mean \pm SD. All statistical analyses were performed using GraphPad Prism software (version 7). The statistically significant differences between two groups were determined using Student's $t$-test. The correlations between the MSC-exo concentration and total radiant efficiency (Figure 2B) were analyzed by Pearson's correlation coefficients. A P-value less than 0.05 was considered statistically significant.

\section{A}

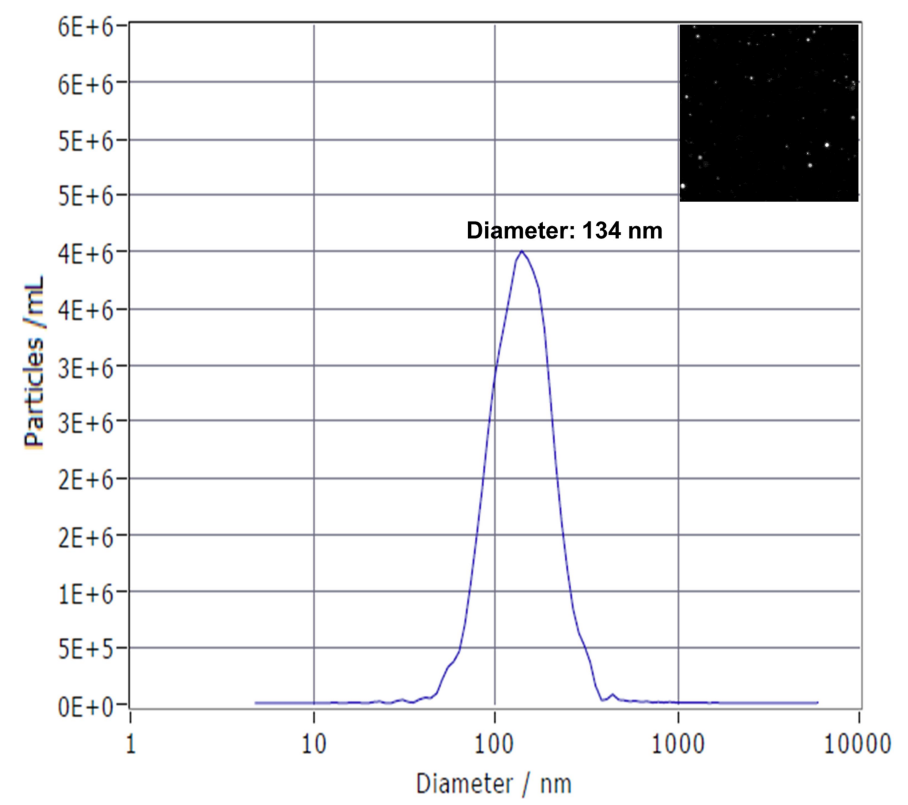

B

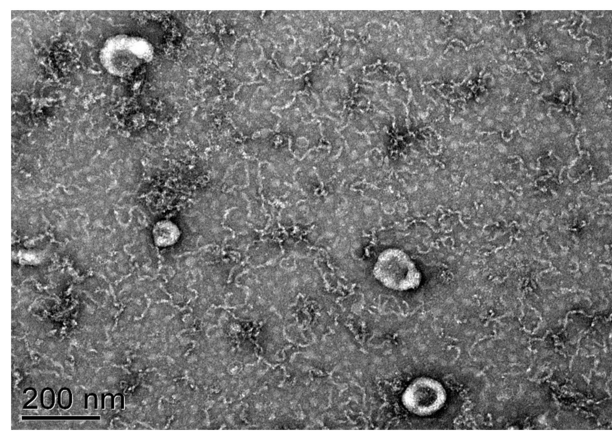

C

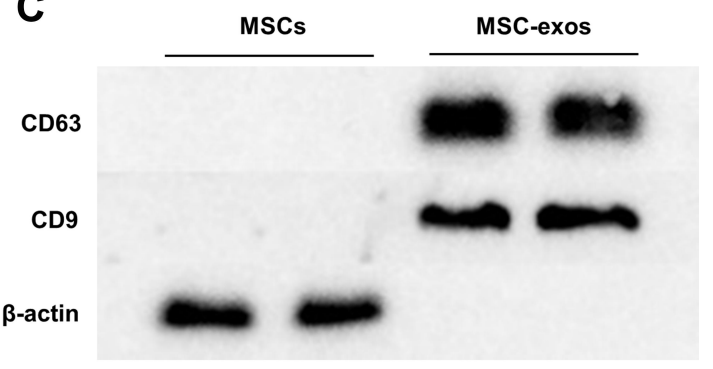

Figure I Exosomes collected from MSC-conditioned medium were characterized by NTA, TEM and Western blotting. (A) NTA of MSC-exo size. (B) Representative TEM image of MSC-exos. Scale bar=200 nm. (C) The expression of the tetraspanins CD9 and CD63 in MSCs and MSC-exos was determined by Western blotting. 
A

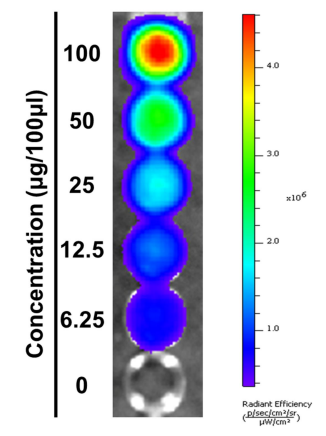

B

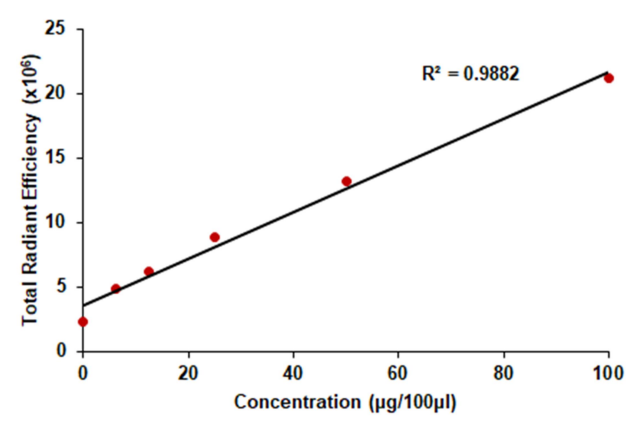

D
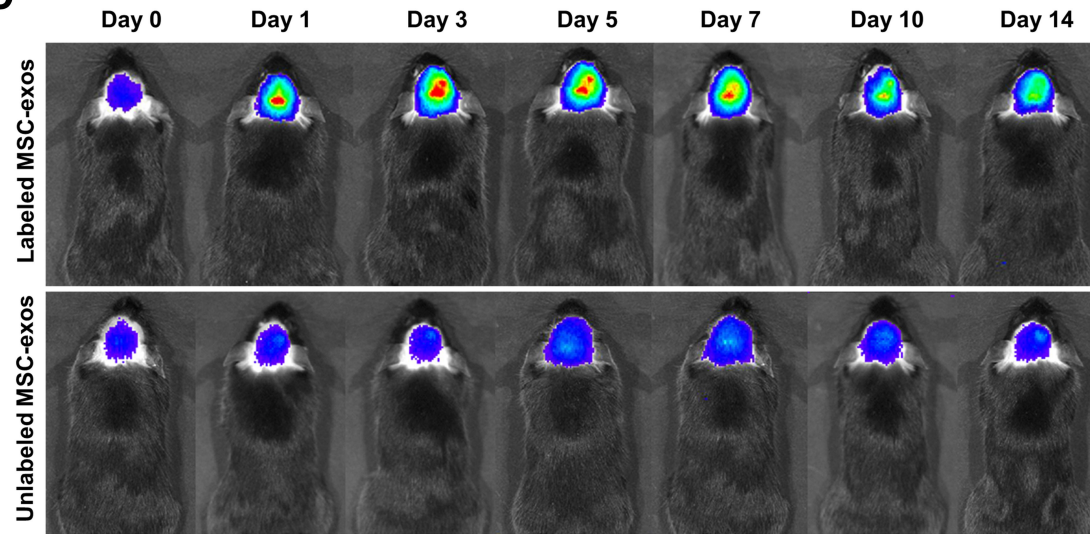

$\mathbf{F}$

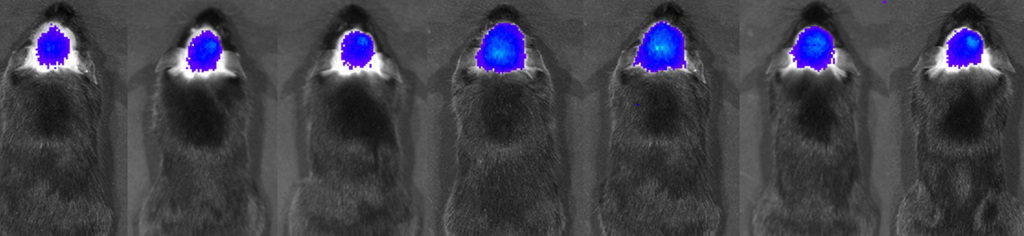

G

C

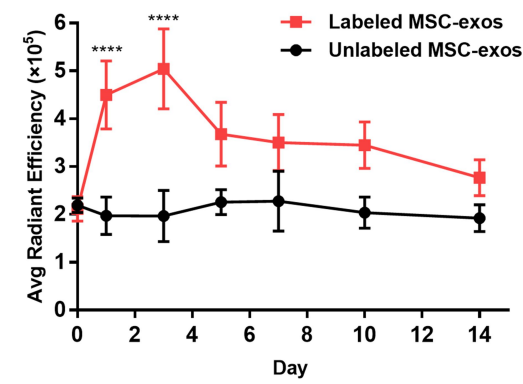

E

E Unlabeled Labeled MSC-exos MSC-exos

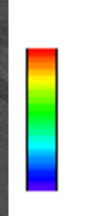
ํㅗㅀ
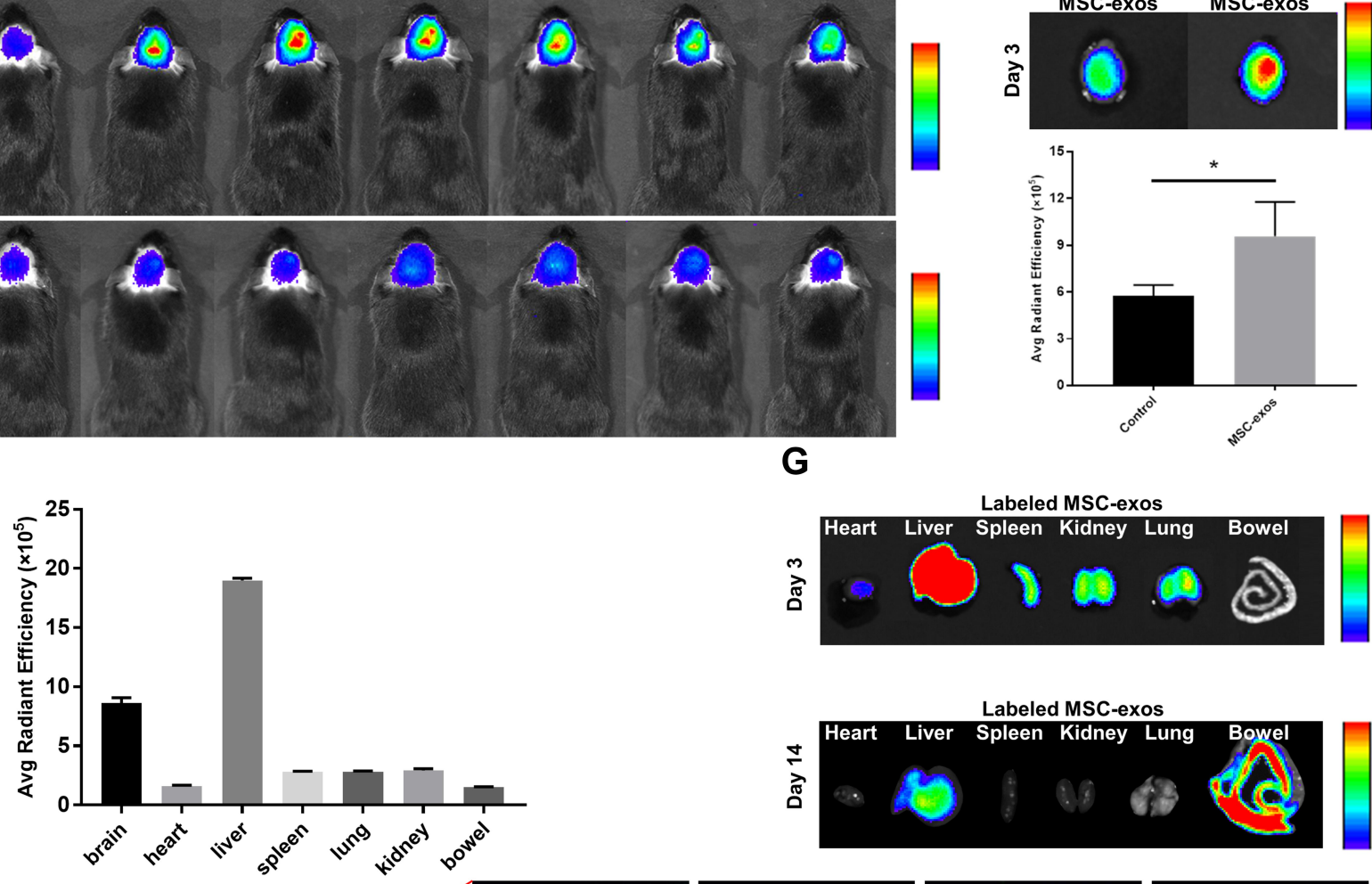

H
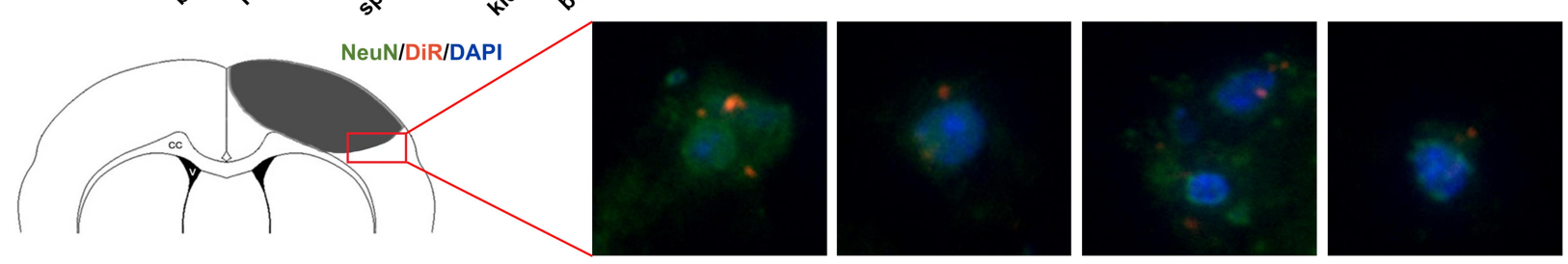

Figure 2 DiR-labeled MSC-exos homed to ischemic foci after stroke. (A) NIRFI of DiR-labeled MSC-exos after ultracentrifugation from free DiR solution. (B) Linear correlation between the fluorescence signal intensity and MSC-exo concentration. (C) The average radiant efficiency based on NIRF images on days I and 3 in the DiR-labeled MSC-exo-treated group was significantly increased compared with that in the unlabeled MSC-exo-treated group ( $n=6-8 /$ group; ***** $<0.000 \mathrm{I})$. (D) Representative NIRF images of ischemic stroke brains from the DiR-labeled group MSC-exo-treated and unlabeled MSC-exo-treated group. (E) Above: representative ex vivo NIRF images of brains from the DiR-labeled MSC-exo-treated group and unlabeled MSC-exo-treated group on day 3. Below: Quantification of the average radiant efficiency based on NIRF images of ex vivo brains on day 3 in the two groups ( $n=3-4 /$ group; ${ }^{*}$ < 0.05 ). (F) The average radiant efficiency based on NIRF images of isolated organs from the DiR-labeled MSC-exo-treated group on day 3. (G) Representative NIRF image of tissues from the DiR-labeled MSC-exo-treated group on days 3 and I4. (H) Representative immunofluorescence images of neurons in the peri-infarct area in the DiR-labeled MSC-exotreated group on day $3, \times 400$.

\section{Results}

\section{Exosome Characterization}

We used three conventional methods, namely, NTA, TEM and Western blotting, to characterize the exosomes. NTA and
TEM showed that the MSC-exos exhibited a typical morphology and size (134 nm) (Figure 1A and B). The expression of CD63 and CD9 (specific markers of exosomes) was verified by a Western blot analysis (Figure 1C). 


\section{DiR-Labeled Exosomes}

To confirm whether we could track the MSC-exos by fluorescence imaging and whether the fluorescence signal intensity could reflect the concentration of the MSC-exos, DiR-labeled MSC-exos were detected by in vitro fluorescence imaging. The results showed that the fluorescence signal intensity was highly linearly correlated with the MSC-exo concentration (Figure 2A and B).

\section{MSC-Exos Homed to Ischemic Foci After Stroke}

After intravenously injecting the MSC-exos, the signals in the ischemic foci were captured in vivo by NIRFI every other day. The results showed that the DiR-labeled MSCexo group exhibited an enhanced fluorescence signal intensity in the ischemic areas in vivo (Figure $2 \mathrm{C}$ and D). The average radiant efficiency of the DiR-labeled MSC-exo group was significantly higher than that of the unlabeled MSC-exo group on day 1 and day 3 (Figure 2C). As determined based on the NIRF images, the average radiant efficiency peaked on day 3. A similar enhancement was also observed in the ex vivo brain (Figure 2E). In addition, the ex vivo NIRF images showed an obvious accumulation of the MSC-exo signal in the liver, and on day 14, the signal enhancement was observed in the bowel (Figure $2 \mathrm{~F}$ and $\mathrm{G}$ ), indicating that the exogenous MSC-exos were mainly excreted through the liver to the bowel.

Brain sections were collected, and immunofluorescence staining was conducted. The results demonstrated that on day 3, the NeuN signal was colocalized with the DiR-labeled MSC-exo signal in the ischemic border zone (IBZ) in the mice treated with MSC-exos (Figure 2H), indicating that some injected MSC-exos were taken up by neurons.

\section{MSC-Exos Decreased the Infarct Volume After Stroke}

The infarcted areas in the murine stroke models showed high intensity signals on T2-weighted images on day 1, day 7 and day 14 (Figure 3A). The infarct volume decreased over time in both the MSC-exo-treated and saline-treated groups, but the delta infarct volume on day 14 was significantly increased as a result of the exogenous MSC-exos, which may have led to better functional outcomes (Figure 3B and C).

\section{MSC-Exos Improved Neurological \\ Functional Recovery After Stroke}

As determined by the mNSS test, the neurological outcomes were significantly improved in the MSC-exo-treated group on day 14 (Figure 3D). Similarly, in the foot-fault test, which was used to evaluate forelimb deficits, the MSC-exo-treated group showed a significant decrease in the number of foot faults on day 14 after stroke (Figure 3E).

\section{MSC-Exos Increased Angiogenesis and Neurogenesis After Stroke}

To investigate the pathophysiological basis underlying the improvements in functional recovery induced by the MSCexos, we used immunohistochemistry and a Western blot analysis. We found that the microvessel density (MVD) along the IBZ was significantly improved in the MSC-exotreated group (Figure 4A). In addition, the exogenous MSCexos significantly increased the number of DCX-positive cells (immature progenitor neurons) and Ki67-positive cells (proliferating cells) in the subventricular zone (SVZ) on day 14 (Figure 4B and C). The Western blot analysis showed that the expression of BDNF, which is crucial for the survival of neurons, was increased in the ipsilateral hemisphere of the mice after the MSC-exo injection, although the difference was not statistically significant (Figure 5). Based on these findings, the MSC-exos promoted angiogenesis and neurogenesis in the mice following stroke.

\section{MSC-Exos Increased White Matter Recovery After Stroke}

On day 14, the FA value, which was determined by DTI, was enhanced in the ipsilateral internal capsule (IC) in the MSCexo-treated group (Figure 6A and B). Significant increases in the fiber count and length in the ipsilateral IC were also observed after the MSC-exo infusion (Figure 6A, C and D). Subsequently, the immunohistochemical staining of the mouse brains revealed that the MBP levels in the ipsilateral IC were significantly increased after the MSC-exo injection on day 14, which is consistent with the DTI results (Figure 6E).

\section{MSC-Exos Altered Cytokines After Stroke}

The expression of specific pro-inflammatory cytokines in the MSC-exo-treated and control groups was determined by a Western blot analysis. Lower levels of IL- $1 \beta$ and tumor necrosis factor- $\alpha$ (TNF- $\alpha)$ were observed as 
A
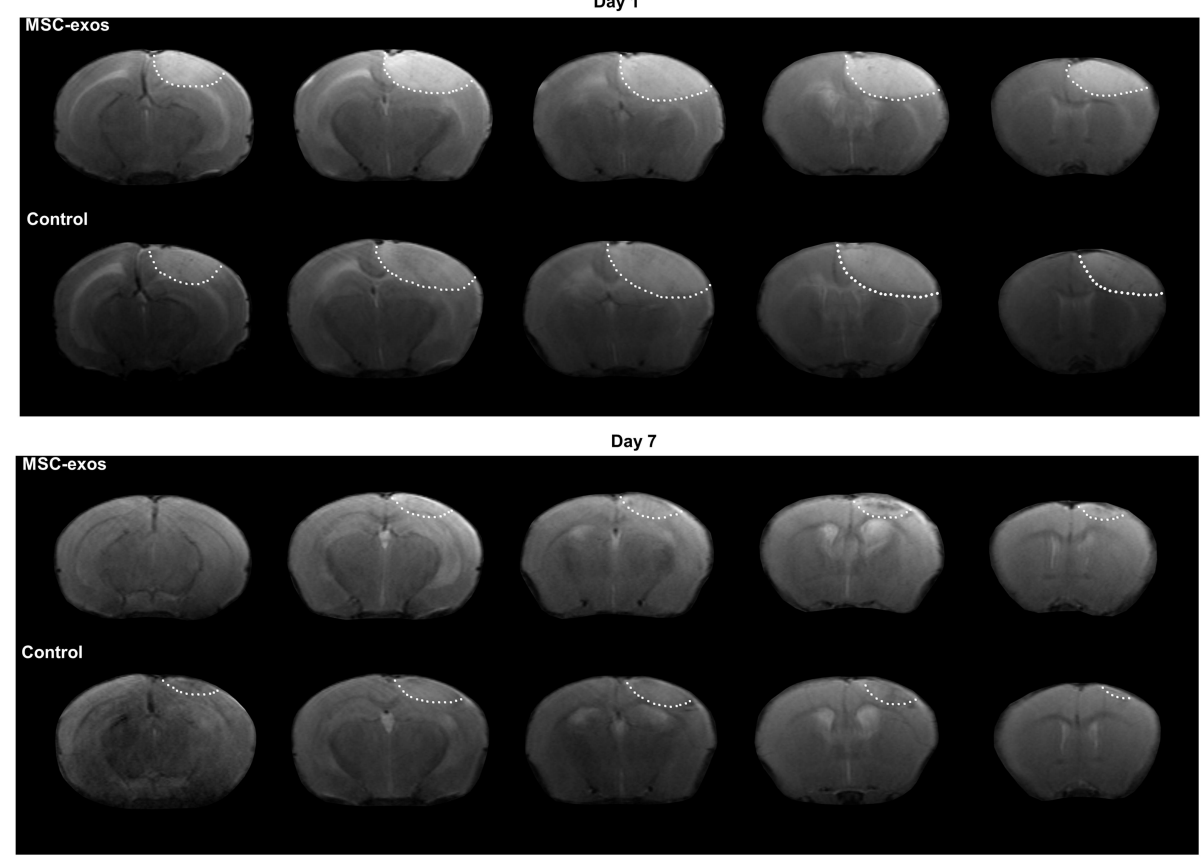

Day 14

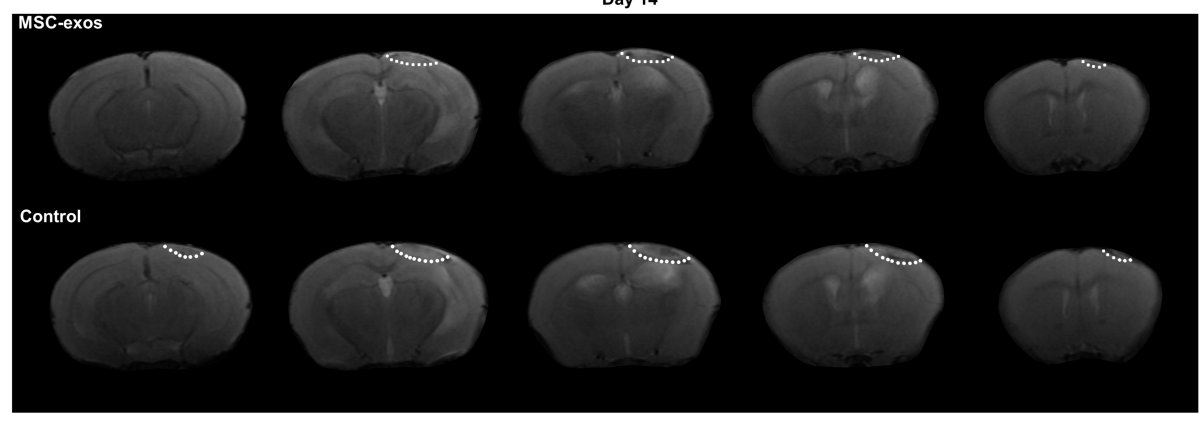

B

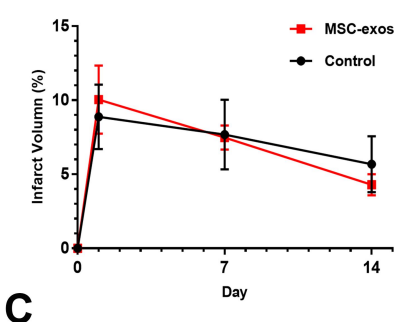

C

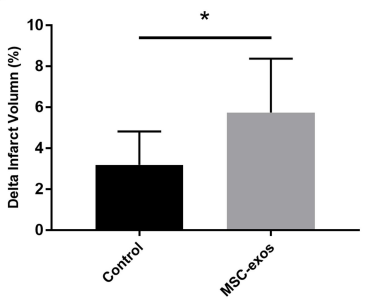

D

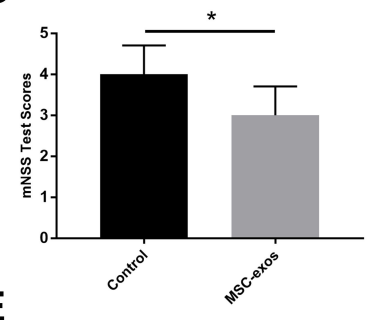

E

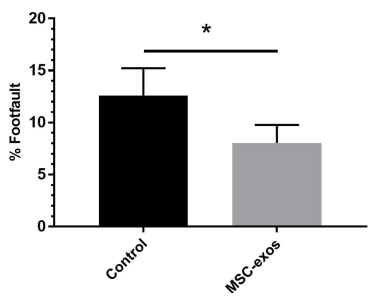

Figure 3 Exogenous MSC-exos reduced the infarct volume and improved functional recovery after ischemic stroke. (A) Representative infarct volume based on T2weighted images. (B) Quantification of the infarct volume in each group on days I, 7, and I4 after stroke. (C) Quantification of the delta infarct volume on day I4 in the two groups. (D) Modified neurological severity scores. (E) Foot-fault test results. $n=6-8 /$ group. ${ }^{*} p<0.05$.

a result of the exogenous MSC-exos (Figure 7A and B), and the expression of IL- $1 \beta$ significantly differed between the two groups. Thus, similar to parent MSCs, MSC-exos may suppress the inflammatory response in the brain after stroke.

\section{Discussion}

Many studies have found that MSCs have a prominent therapeutic potential in regenerative medicine by integrating into the damaged lesion and acting as storage for restorative molecules. The pathophysiology underlying MSC homing mainly consist of the following five components: 1) initial tethering by selectins, 2) activation by cytokines, 3) arrest by integrins, 4) transmigration using matrix remodelers, and 5) extravascular migration toward chemokine gradients. $^{33}$ However, compared with the injection quantity, only a small percentage of exogenous MSCs arrive in the injured brain. Nevertheless, the therapeutic outcomes of MSCs have been confirmed by many studies. $^{34-37}$ Some reports have revealed that MSCs release exosomes in the circulatory system to exert their effects rather than directly homing to ischemic regions. ${ }^{38}$ In addition, currently, it is widely believed that exosomes can cross the BBB during central nervous system inflammation. ${ }^{39}$ Overall, these results suggest that it is possible for intravenously injected MSC-exos to migrate into the injured brain and act as therapeutic agents after stroke.

MSC-exos have received increasing attention because they are regarded as crucial communicators that transmit biological information and may have the potential to replace their corresponding parental MSCs. ${ }^{40}$ MSC-exos are 

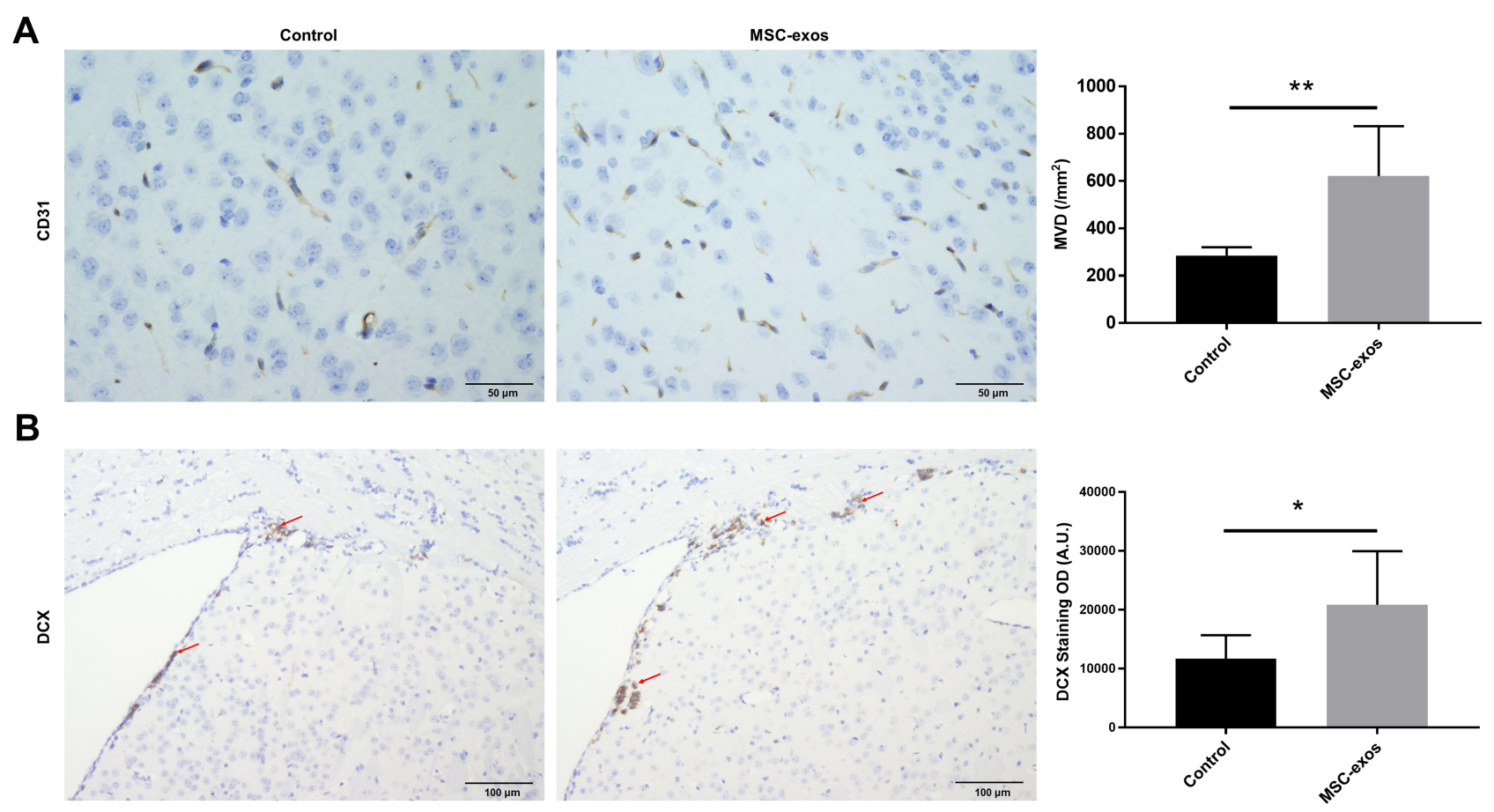

C
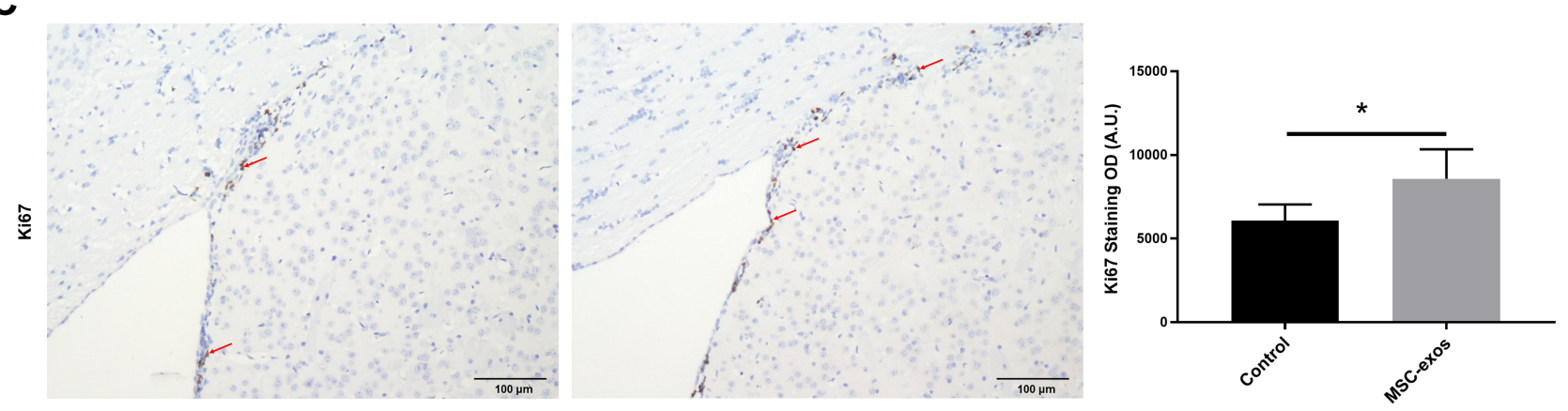

Figure 4 Exogenous MSC-exos increased angiogenesis and neurogenesis after stroke. (A) Representative images of CD3I staining in the IBZ and quantitative data of MVD on day 14. (B) Representative images of DCX staining in the SVZ and quantitative data on day 14. (C) Representative images of Ki67 staining in the SVZ and quantitative data on day 14. $n=8$ - 10 /group. ${ }^{p}<0.05$, ** $p<0.01$.

considered to have specific therapeutic effects on damage in various organs. ${ }^{15-20}$ In particular, MSC-exos are among the most popular candidates for the treatment of central nervous system diseases, such as Parkinson's disease, Alzheimer's disease, and cerebrovascular disease, due to their easy access to the BBB. ${ }^{41}$ Some studies have shown that MSC-exos can increase neurovascular plasticity, axonal and neurite remodeling, angiogenesis and functional recovery. ${ }^{16,24,25,42}$ However, to date, the migration of exosomes in ischemic stroke has not been thoroughly investigated. In vivo imaging of exosomes may provide novel evidence concerning the mechanism underlying the therapeutic effects of MSC-exos in ischemic stroke; thus, it is important to elucidate the regenerative potential of MSC-exos in ischemic stroke. Understanding the migration and homing patterns of exosomes may be beneficial for the application of exosomes. Here, we quantitatively and longitudinally (over weeks) observed the homing of MSC-exos in ischemic stroke mice using a DiR-based imaging method. DiR (1,1-dioctadecyl3,3,3,3-tetramethylindotricarbocyanine-iodide) is a lipophilic dye with a fluorescence emission maximum of the near infrared that has high tissue penetration and low autofluorescence. ${ }^{43-45} \mathrm{DiR}$ has been widely used to label cells for in vivo imaging for a long time. Carlson et al tracked the proliferation of DiR-labeled hematopoietic stem/progenitor cells and mature T cells in mice up to $135 \mathrm{~h} .{ }^{46}$ Kuffler DP suggested that the half-life of DiR in vivo is approximately 4 weeks. ${ }^{47}$ These studies revealed that DiR could be used for long-term and repeated imaging in vivo. Recently, some novel nanocarriers, such as liposomes, exosomes, micelles, 

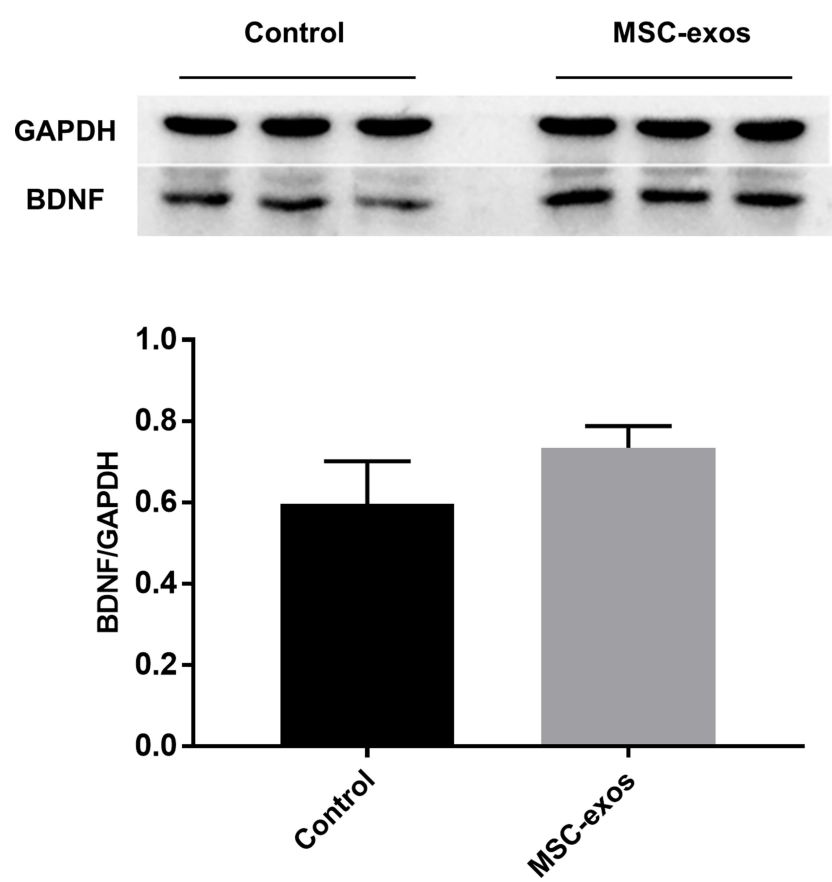

Figure 5 Exogenous MSC-exos increased BDNF expression in the ischemic brain. Western blot analysis of BDNF expression in the ipsilateral hemisphere and quantitative data on day 14. $n=3$ /group.

and polymeric nanoparticles, have been investigated using DiR labeling and imaging. ${ }^{48-51}$

By combining gold nanoparticle labeling and CT imaging, a previous study demonstrated that MSC-exos displayed a significant tendency of migrating to specific lesion sites and distinct distribution patterns in various brain pathologies, including ischemic stroke, Parkinson's disease, Alzheimer's disease, and autism spectrum. ${ }^{52}$ The accumulation of MSC-exos in ischemic stroke increased over time and persisted up to $96 \mathrm{~h}^{52}$

In our study, we labeled MSC-exos by DiR, prolonged the observation time and found that the MSC-exos were able to migrate into the infarcted brain and remain there for up to 14 days. Therefore, exosomes are a feasible option for delivery to the brain, further supporting earlier findings by other researchers who took advantage of the ability of exosomes to deliver cargo to treat central nervous system diseases. ${ }^{17}$ The mechanisms underlying MSC-exo migration are still unclear. It is reasonable to infer that MSC-exos possess the same extracellular matrix binding proteins and surface receptors as MSCs, resulting in homing abilities. Many reports have shown that the chemokine receptors expressed by MSCs, such as CXCR4, may play a key role in recruiting MSCs to the host brain. ${ }^{53,54}$

In the current study, we found that some MSC-exos applied by intravenous injection could target ischemic lesions after stroke through their natural homing capability. In addition, we found that in contrast to MSCs, the MSC-exos did not accumulate in the lung as previously reported. ${ }^{55}$ However, the MSC-exos could not escape phagocytosis in the liver. By detecting DiR-labeled MSC-exos in vivo for two weeks, we discovered that in addition to the target tissues, the liver showed a high MSC-exo signal throughout the observation period. Therefore, developing approaches to reduce exosome accumulation in the liver and allow exosomes to reach target organs is worthy of consideration. Further studies may focus on membrane modification to promote MSC-exo homing to the brain.

Moreover, our study investigated the therapeutic capability of MSC-exos in ischemic stroke and showed that similar to parental MSCs, MSC-exos may participate in various neuroprotective activities, including promoting functional recovery, neurogenesis, and angiogenesis, after stroke. These results are consistent with previously published studies. ${ }^{16,23,24,26}$

Additionally, we investigated the effect of MSC-exos on inflammation after stroke. Ischemic stroke is always accompanied by local neuroinflammation. Long-term inflammatory processes lead to the destruction of homeostasis in the brain, thus further aggravating the effects of stroke. Both preclinical and clinical studies have reported that MSC-exos exert anti-inflammatory effects. ${ }^{56}$ Similarly, our study shows that the expression of the proinflammatory cytokine IL-1 $\beta$ in the mice injected with the MSC-exos was obviously lower than that in the control mice. Another pro-inflammatory factor, ie, TNF- $\alpha$, showed a decreasing tendency, although no significant difference was found between the two groups. Subtle or complex inflammatory effects are involved in many events after ischemic stroke. The suppression of inflammation by MSC-exos can result in a better microenvironment for neuronal repair and regeneration.

Although many studies have confirmed the functional roles of MSC-exos in the restoration of the ischemic hemisphere by conducting behavioral analyses or histological analyses of tissues, few studies integrated DTI, which can directly and noninvasively demonstrate the recovery of neuronal fiber bundles. DTI is widely used to evaluate white matter changes in real-time in stroke patients. The IC is an indispensable component of the corticospinal tract (CST) that governs motor function. DTI showed that the MSC-exo treatment enhanced the FA values, indicating higher anisotropy of axons in the ipsilateral IC of ischemic mice. Fiber tracing revealed an 


\section{A}
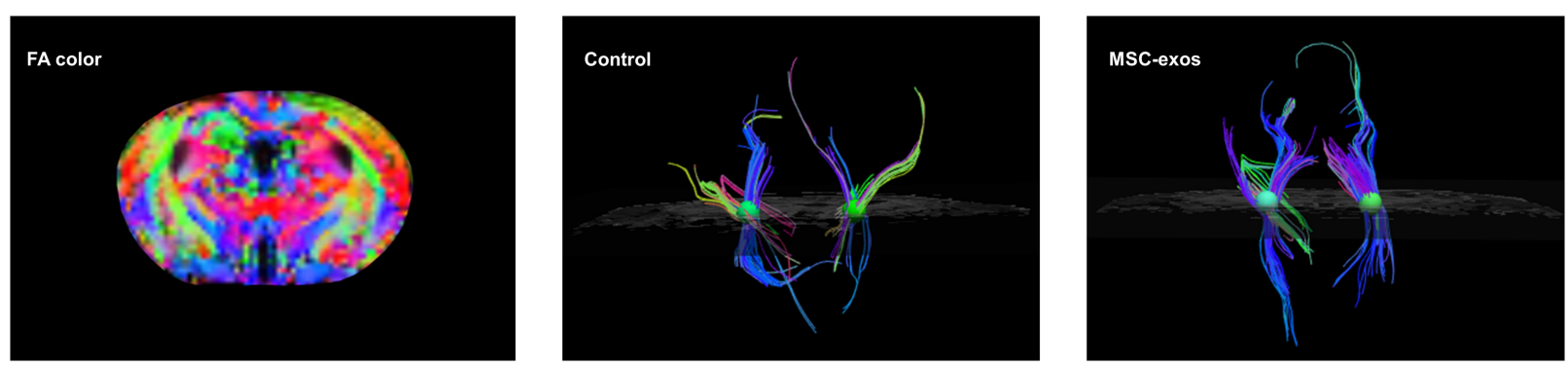

B

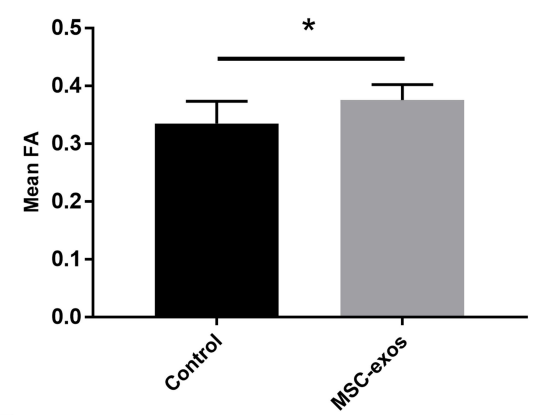

$\mathbf{E}$

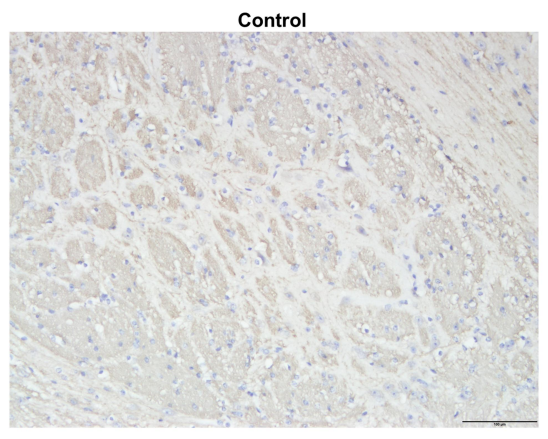

C

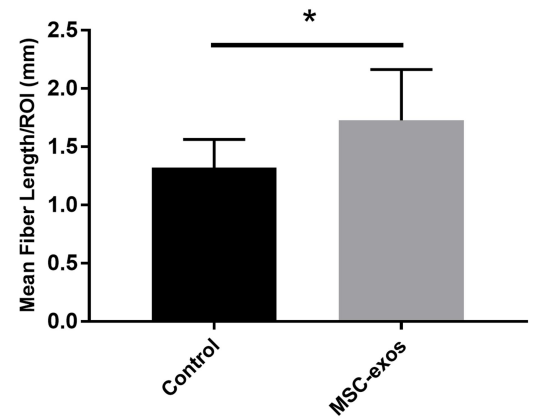

MSC-exos

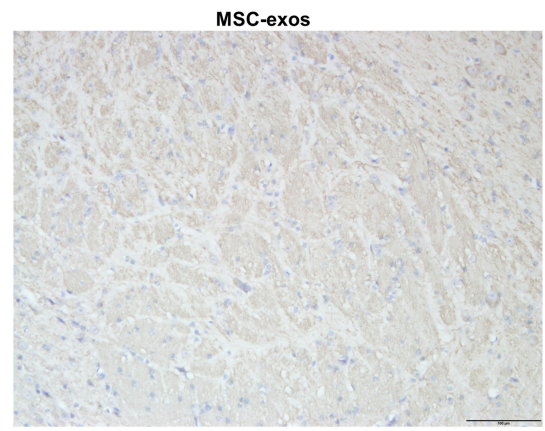

D
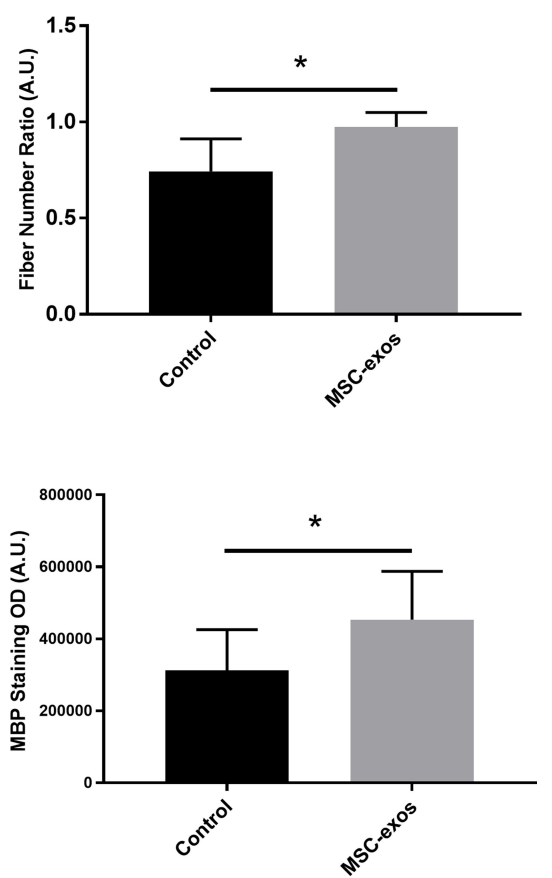

Figure 6 Exogenous MSC-exos promoted white matter recovery after stroke. (A) Fiber tracking in the IC using DTI. (B) FA values in the ipsilesional IC in the MSC-exotreated group and control group. (C) Fiber length in the ipsilesional IC. (D) Fiber number ratio (fiber number in the ipsilesional IC: fiber number in the contralesional IC). (E) Representative images of MBP staining in the ipsilesional IC and quantitative data on day I4. $n=6-8 /$ group. ${ }^{*} p<0.05$.

improvement in both the fiber number and length in the ipsilateral IC. These results all demonstrate that the MSC-exo treatment enhanced white matter recovery after stroke, which is consistent with the histological results. Thus, DTI may be an effective technique for the noninvasive assessment of the level of axonal recovery.

Compared with MSCs, exosomes are unlikely to obstruct blood vessels due to their nano-level size; thus, exosomes have an extremely low risk of embolism. ${ }^{12}$ Moreover, exosomes are more stable and easier to preserve than living cells because they cannot differentiate and proliferate. Similar to parental MSCs, MSC-exos are immunosuppressive and could be used for allogenic administration. ${ }^{57}$ Therefore, the clinical application of MSC-exos appears promising.

\section{Limitations}

This study lays a theoretical foundation for the clinical application of MSC-exos mainly by focusing on describing the phenomenon in which MSC-exos could home to the ischemic brain. However, most injected MSC-exos were trapped in the liver. Further studies need to be performed to improve the homing efficiency of MSC-exos and use of MSC-exos as nanocarriers for drug delivery. Additionally, we only focused on whether the MSC-exos were taken up by neurons and exerted their influence but ignored other cells in the infract area, such as microglia and astrocytes. Future studies should focus on the potential pathophysiological process of these cells after exosome injection. 
A
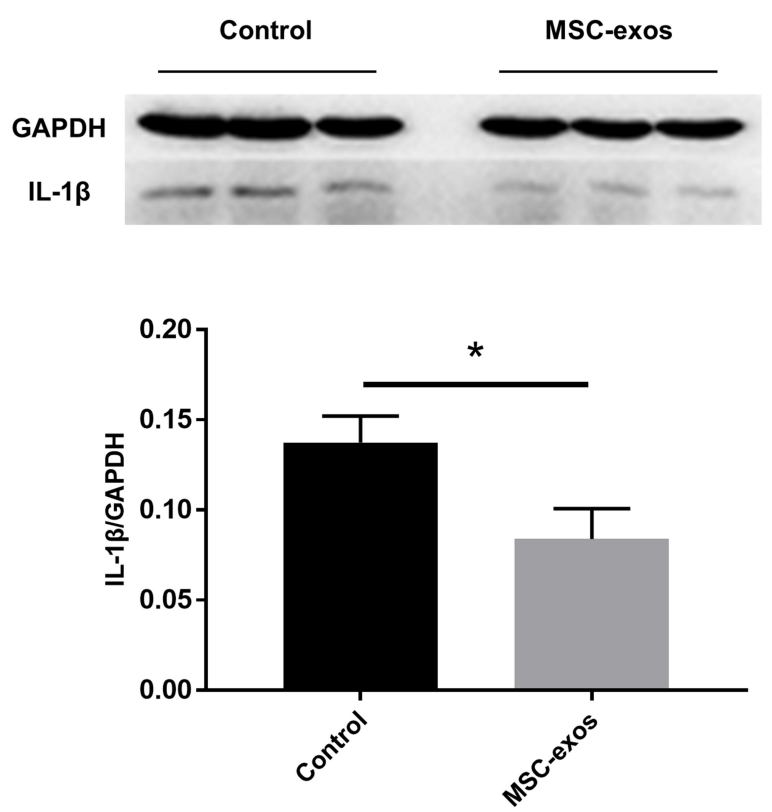

B
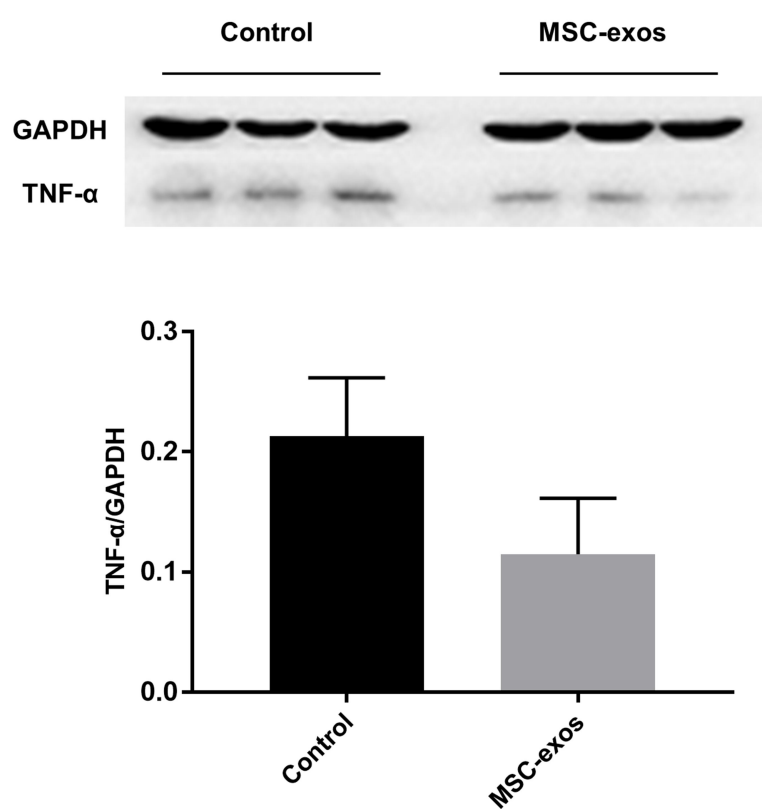

Figure 7 Exogenous MSC-exos reduced inflammation in the ischemic brain on day I4. (A) Western blot analysis of IL-I $\beta$ expression in the ipsilateral hemisphere and quantitative data. (B) Western blot analysis of TNF- $\alpha$ expression in the ipsilateral hemisphere and quantitative data. $n=3 / g r o u p . ~ * p<0.05$.

Despite the positive outlook of MSC-exos, some key points are notable. First, each exosome isolation and purification approach has advantages and disadvantages. Due to the lack of a unified method, directly comparing different findings is difficult. Second, the dosage regimens of exosome applications remain an uncertain factor that may affect treatment outcomes. To date, a standard dosage regimen is lacking for both animal experiments and clinical trials. Therefore, more research concerning exosomes is needed.

\section{Conclusion}

We can draw the following conclusions based on our study: 1) MSC-exos can effectively migrate into ischemic lesions and fuse with neurons after intravenous injection; 2) MSC-exos promote neurogenesis and angiogenesis after stroke; 3) the DTI results were highly consistent with the pathological results, and DTI can be used to directly demonstrate white matter remodeling; and 4) to some extent, inflammation triggered by ischemic stroke is relieved by MSC-exo treatment.

\section{Abbreviations}

MSC-exos, mesenchymal stem cell-derived exosomes; NIRF, near-infrared fluorescence; MR, magnetic resonance; DTI, diffusion tensor imaging; FA, fractional anisotropy; rt-PA, recombinant tissue plasminogen activator; $\mathrm{BBB}$, blood-brain barrier; MSCs, mesenchymal stem cells; MRI, magnetic resonance imaging; FBS, fetal bovine serum; TEM, transmission electron microscopy; NTA, nanoparticle tracking analysis; NIRFI, near-infrared fluorescence imaging; IACUC, Institutional Animal Care and Use Committee; mNSS, modified neurological severity score; T2WI, T2-weighted imaging; FOV, field of view; IC, internal capsule; MBP, maltose-binding protein; DCX, doublecortin; BDNF, brainderived neurotrophic factor; MVD, microvessel density; IBZ, ischemic border zone; SVZ, subventricular zone; TNF$\alpha$, tumor necrosis factor- $\alpha$; CST, corticospinal tract.

\section{Funding}

This research was supported by National Natural Science Foundation of China (NSFC, No. 81601547, 81830053 and 81525014), the National Key Research and Development Program of China (2017YFA0104302), the Natural Science Foundation of Jiangsu Province (BK20160703, BK20180377), Jiangsu Provincial Medical Youth Talent (NRC2016823), and Science Foundation for Creative Research Groups of the Ministry of Science and Technology of China (No. 6290002012).

\section{Disclosure}

The authors have no conflicts of interest in this work to report. 


\section{References}

1. Hankey GJ. Stroke. Lancet. 2017;389(10069):641-654. doi:10.1016/ S0140-6736(16)30962-X

2. GBD 2016 Stroke Collaborators. Global, regional, and national burden of stroke, 1990-2016: a systematic analysis for the global burden of disease study 2016. Lancet Neurol. 2019;18(5):439-458.

3. Lv J, Hu W, Yang Z, et al. Focusing on claudin-5: A promising candidate in the regulation of BBB to treat ischemic stroke. Prog Neurobiol. 2018;161:79-96. doi:10.1016/j.pneurobio.2017.12.001

4. Pandian JD, Gall SL, Kate MP, et al. Prevention of stroke: a global perspective. Lancet. 2018;392(10154):1269-1278.

5. Hacke W, Kaste M, Bluhmki E, et al. Thrombolysis with alteplase 3 to 4.5 hours after acute ischemic stroke. $N$ Engl J Med. 2008;359 (13):1317-1329. doi:10.1056/NEJMoa0804656

6. Jauch EC, Saver JL, Adams HP Jr, et al. Guidelines for the early management of patients with acute ischemic stroke: a guideline for healthcare professionals from the american heart association/american stroke association. Stroke. 2013;44(3):870-947.

7. Bluhmki E, Chamorro A, Davalos A, et al. Stroke treatment with alteplase given $3 \cdot 0-4 \cdot 5 \mathrm{~h}$ after onset of acute ischaemic stroke (ECASS III): additional outcomes and subgroup analysis of a randomised controlled trial. Lancet Neurol. 2009;8(12):1095-1102. doi:10.1016/S1474-4422(09)70264-9

8. Vanderkerken K, Asosingh K, Croucher P, Van Camp B. Multiple myeloma biology: lessons from the 5TMM models. Immunol Rev. 2003;194:196-206. doi:10.1034/j.1600-065X.2003.00035.x

9. Chen J, Li Y, Wang L, et al. Therapeutic benefit of intravenous administration of bone marrow stromal cells after cerebral ischemia in rats. Stroke. 2001;32(4):1005-1011. doi:10.1161/01.STR.32.4. 1005

10. Zhu W, Xu W, Jiang R, et al. Mesenchymal stem cells derived from bone marrow favor tumor cell growth in vivo. Exp Mol Pathol. 2006;80(3):267-274. doi:10.1016/j.yexmp.2005.07.004

11. Fierro FA, Sierralta WD, Epuñan MJ, et al. Marrow-derived mesenchymal stem cells: role in epithelial tumor cell determination. Clin Exp Metastasis. 2004;21(4):313-319. doi:10.1023/B:CLIN.000 0046130.79363 .33

12. Janowski M, Lyczek A, Engels C, et al. Cell size and velocity of injection are major determinants of the safety of intracarotid stem cell transplantation. J Cereb Blood Flow Metab. 2013;33:921-927.

13. Yeo RW, Lai RC, Zhang B, et al. Mesenchymal stem cell: an efficient mass producer of exosomes for drug delivery. Adv Drug Deliv Rev. 2013;65(3):336-341. doi:10.1016/j.addr.2012.07.001

14. Altanerova U, Babincova M, Babinec P, et al. Human mesenchymal stem cell-derived iron oxide exosomes allow targeted ablation of tumor cells via magnetic hyperthermia. Int $J$ Nanomedicine. 2017;27(12):7923-7936. doi:10.2147/IJN.S145096

15. Camussi G, Deregibus MC, Cantaluppi V. Role of stem-cell-derived microvesicles in the paracrine action of stem cells. Biochem Soc Trans. 2013;41(1):283-287. doi:10.1042/BST20120192

16. Xin H, Li Y, Cui Y, Yang JJ, Zhang ZG, Chopp M. Systemic administration of exosomes released from mesenchymal stromal cells promote functional recovery and neurovascular plasticity after stroke in rats. J Cereb Blood Flow Metab. 2013;33(11):1711-1715. doi: $10.1038 /$ jcbfm. 2013.152

17. Bruno S, Grange C, Deregibus MC, et al. Mesenchymal stem cell-derived microvesicles protect against acute tubular injury. $J \mathrm{Am}$ Soc Nephrol. 2009;20(5):1053-1067. doi:10.1681/ASN.2008070798

18. Lai RC, Arslan F, Lee MM, et al. Exosome secreted by MSC reduces myocardial ischemia/reperfusion injury. Stem Cell Res. 2010;4 (3):214-222. doi:10.1016/j.scr.2009.12.003

19. Hu GW, Li Q, Niu X, et al. Exosomes secreted by human-induced pluripotent stem cell-derived mesenchymal stem cells attenuate limb ischemia by promoting angiogenesis in mice. Stem Cell Res Ther. 2015;10(6):10.
20. Wei H, Chen J, Wang S, et al. A nanodrug consisting of doxorubicin and exosome derived from mesenchymal stem cells for osteosarcoma treatment in vitro. Int $J$ Nanomedicine. 2019;1(14):8603-8610. doi:10.2147/IJN.S218988

21. Vakhshiteh F, Atyabi F, Ostad SN. Mesenchymal stem cell exosomes: a two-edged sword in cancer therapy. Int J Nanomedicine. 2019;23 (14):2847-2859. doi:10.2147/IJN.S200036

22. Camussi G, Deregibus MC, Bruno S, Grange C, Fonsato V, Tetta C. Exosome/microvesicle-mediated epigenetic reprogramming of cells. Am J Cancer Res. 2011;1(1):98-110.

23. Li Y, Cheng Q, Hu G, et al. Extracellular vesicles in mesenchymal stromal cells: a novel therapeutic strategy for stroke. Exp Ther Med. 2018;15(5):4067-4079.

24. Xin H, Katakowski M, Wang F, et al. MicroRNA cluster miR-1792 cluster in exosomes enhance neuroplasticity and functional recovery after stroke in rats. Stroke. 2017;48(3):747-753.

25. Xin H, Li Y, Liu Z, et al. MiR-133b promotes neural plasticity and functional recovery after treatment of stroke with multipotent mesenchymal stromal cells in rats via transfer of exosome-enriched extracellular particles. Stem Cells. 2013;31(12):2737-2746. doi:10.1002/stem.1409

26. Liang X, Zhang L, Wang S, Han Q, Zhao RC. Exosomes secreted by mesenchymal stem cells promote endothelial cell angiogenesis by transferring miR-125a. J Cell Sci. 2016;129(11):2182-2189. doi: $10.1242 /$ jcs. 170373

27. Moon GJ, Sung JH, Kim DH, et al. Application of mesenchymal stem cell-derived extracellular vesicles for stroke: biodistribution and microRNA study. Transl Stroke Res. 2019;10(5):509-521. doi:10.1007/s12975-018-0668-1

28. Pfefferbaum A, Sullivan EV. Microstructural but not macrostructural disruption of white matter in women with chronic alcoholism. Neuroimage. 2002;15(3):708-718.

29. Thomason ME, Thompson PM. Diffusion imaging, white matter, and psychopathology. Апnи Rev Clin Psychol. 2011;7:63-85.

30. Meng SS, Xu XP, Chang W, et al. LincRNA-p21 promotes mesenchymal stem cell migration capacity and survival through hypoxic preconditioning. Stem Cell Res Ther. 2018;9(1):280.

31. Clarkson AN, Huang BS, Macisaac SE, Mody I, Carmichael ST. Reducing excessive GABA-mediated tonic inhibition promotes functional recovery after stroke. Nature. 2010;468(7321):305-309.

32. Watanabe T, Okuda Y, Nonoguchi N, et al. Postischemic intraventricular administration of FGF-2 expressing adenoviral vectors improves neurologic outcome and reduces infarct volume after transient focal cerebral ischemia in rats. J Cereb Blood Flow Metab. 2004;24(11):1205-13.

33. Cui X, Chopp M, Zacharek A, Cui Y, Roberts C, Chen J. The neurorestorative benefit of GW3965 treatment of stroke in mice. Stroke. 2013;44(1):153-161.

34. Karp JM, Leng Teo GS. Mesenchymal stem cell homing: the devil is in the details. Cell Stem Cell. 2009;4(3):206-216. doi:10.1016/j. stem.2009.02.001

35. Rosenblum S, Wang N, Smith TN, et al. Timing of intra-arterial neural stem cell transplantation after hypoxia-ischemia influences cell engraftment, survival, and differentiation. Stroke. 2012;43 (6):1624-1631. doi:10.1161/STROKEAHA.111.637884

36. Huang P, Gebhart N, Richelson E, Brott TG, Meschia JF, Zubair AC. Mechanism of mesenchymal stem cell induced neuron recovery and anti-inflammation. Cytotherapy. 2014;16 (10):1336-1344.

37. Hsuan YC, Lin CH, Chang CP, Lin MT. Mesenchymal stem cell based treatments for stroke, neural trauma, and heat stroke. Brain Behav. 2016;6(10):e00526. doi:10.1002/brb3.526

38. Iso Y, Spees JL, Serrano C, et al. Multipotent human stromal cells improve cardiac function after myocardial infarction in mice without long-term engraftment. Biochem Biophys Res Commun. 2007;354 (3):700-706. doi:10.1016/j.bbrc.2007.01.045 
39. Preda MB, Rønningen T, Burlacu A, Simionescu M, Moskaug JØ, Valen G. Remote transplantation of mesenchymal stem cells protects the heart against ischemia-reperfusion injury. Stem Cells. 2014;32 (8):2123-2134. doi:10.1002/stem.1687

40. Chen CC, Liu L, Ma F, et al. Elucidation of exosome migration across the blood-brain barrier model in vitro. Cell Mol Bioeng. 2016;9(4):509-529. doi:10.1007/s12195-016-0458-3

41. Doeppner TR, Herz J, Görgens A, et al. Extracellular vesicles improve post-stroke neuroregeneration and prevent postischemic immunosuppression. Stem Cells Transl Med. 2015;4(10):1131-1143. doi:10.5966/sctm.2015-0078

42. Katsuda T, Tsuchiya R, Kosaka N, et al. Human adipose tissue derived mesenchymal stem cells secrete functional neprilysin bound exosomes. Sci Rep. 2013;3:1197. doi:10.1038/srep01197

43. Zhang Y, Chopp M, Liu XS, et al. Exosomes derived from mesenchymal stromal cells promote axonal growth of cortical neurons. Mol Neurobiol. 2017;54(4):2659-2673. doi:10.1007/s12035-016-9851-0

44. Hood JL, San RS, Wickline SA. Exosomes released by melanoma cells prepare sentinel lymph nodes for tumor metastasis. Cancer Res. 2011;71(11):3792-3801.

45. Luo S, Zhang E, Su Y, Cheng T, Shi C. A review of NIR dyes in cancer targeting and imaging. Biomaterials. 2011;32(29):7127-7138. doi:10.1016/j.biomaterials.2011.06.024

46. Verbeek FP, van der Vorst JR, Schaafsma BE, et al. Image-guided hepatopancreatobiliary surgery using near-infrared fluorescent light. J Hepatobiliary Pancreat Sci. 2012;19(6):626-637. doi:10.1007/ s00534-012-0534-6

47. Carlson AL, Fujisaki J, Wu J, et al. Tracking single cells in live animals using a photoconvertible near-infrared cell membrane label. PLoS One. 2013;8(8):e69257. doi:10.1371/journal.pone.0069257

48. Kuffler DP. Long-term survival and sprouting in culture by motoneurons isolated from the spinal cord of adult frogs. J Comp Neurol. 1990;302(4):729-738. doi:10.1002/cne.903020405

49. Gao JQ, Lv Q, Li LM, et al. Glioma targeting and blood-brain barrier penetration by dual-targeting doxorubincin liposomes. Biomaterials. 2013;34(22):5628-5639. doi:10.1016/j.biomaterials.2013.03.097
50. Smyth T, Kullberg M, Malik N, Smith-Jones P, Graner MW, Anchordoquy TJ. Biodistribution and delivery efficiency of unmodified tumor-derived exosomes. J Control Release. 2015;10 (199):145-155. doi:10.1016/j.jconrel.2014.12.013

51. Baumann BC, Kao GD, Mahmud A, et al. Enhancing the efficacy of drug-loaded nanocarriers against brain tumors by targeted radiation therapy. Oncotarget. 2013;4(1):64-79. doi:10.18632/oncotarget.777

52. Li K, Liu H, Gao W, et al. Mulberry-like dual-drug complicated nanocarriers assembled with apogossypolone amphiphilic starch micelles and doxorubicin hyaluronic acid nanoparticles for tumor combination and targeted therapy. Biomaterials. 2015;39:131-144. doi:10.1016/j.biomaterials.2014.10.073

53. Perets N, Betzer O, Shapira R, et al. Golden exosomes selectively target brain pathologies in neurodegenerative and neurodevelopmental disorders. Nano Lett. 2019;19(6):3422-3431. doi:10.1021/acs. nanolett.8b04148

54. Bang OY, Jin KS, Hwang MN, et al. The effect of CXCR4 overexpression on mesenchymal stem cell transplantation in ischemic stroke. Cell Med. 2012;4(2):65-76. doi:10.3727/215517912X647172

55. Sordi V. Mesenchymal stem cell homing capacity. Transplantation. 2009;87(9 Suppl):S425. doi:10.1097/TP.0b013e3181a28533

56. Scarfe L, Taylor A, Sharkey J, et al. Non-invasive imaging reveals conditions that impact distribution and persistence of cells after in vivo administration. Stem Cell Res Ther. 2018;9(1):332. doi:10.1186/s13287-018-1076-x

57. Elahi FM, Farwell DG, Nolta JA, Anderson JD. Preclinical translation of exosomes derived from mesenchymal stem/stromal cells. Stem Cells. 2020;38(1):15-21. doi:10.1002/stem.3061

58. Ankrum JA, Ong JF, Karp JM. Mesenchymal stem cells: immune evasive, not immune privileged. Nat Biotechnol. 2014;32(252):260. doi: $10.1038 /$ nbt. 2816
International Journal of Nanomedicine

\section{Publish your work in this journal}

The International Journal of Nanomedicine is an international, peerreviewed journal focusing on the application of nanotechnology in diagnostics, therapeutics, and drug delivery systems throughout the biomedical field. This journal is indexed on PubMed Central, MedLine, CAS, SciSearch ${ }^{\circledR}$, Current Contents ${ }^{\circledR} /$ Clinical Medicine,
Journal Citation Reports/Science Edition, EMBase, Scopus and the Elsevier Bibliographic databases. The manuscript management system is completely online and includes a very quick and fair peer-review system, which is all easy to use. Visit http://www.dovepress.com/ testimonials.php to read real quotes from published authors. 